\title{
The Role of Ga-68-PSMA PET/CT in the Initial Staging of Prostate Cancer - A Single Center 4 Year Experience
}

\author{
João P Lima $\mathbb{D}^{\prime}$ \\ João Carvalho' \\ Vasco Quaresma' \\ Edgar Tavares-da-Silva (D) \\ Rodolfo Silva ${ }^{2}$ \\ Paulo Azinhais ${ }^{2}$ \\ Gracinda Costa ${ }^{2}$ \\ Arnaldo Figueiredo' \\ 'Urology and Renal Transplantation \\ Department, Coimbra University \\ Hospital Center, Coimbra, Portugal; \\ ${ }^{2}$ Nuclear Medicine Department, Coimbra \\ University Hospital Centre, Coimbra, \\ Portugal
}

Background: Recommended imaging modalities for prostate cancer staging have disappointing sensitivities, whereas [68Ga]-PSMA PET/CT (PET-PSMA) shows promising sensitivities and specificities in the initial management of prostate cancer. Recent studies have revealed that a significant change of management when PET-PSMA was used, with favorable negative predictive values.

Methods: In this retrospective study, we analyzed every PET-PSMA performed in our center for initial staging of intermediate and high-risk prostate cancer. Patients were divided into two groups based on whether imaging modalities other than PET-PSMA were performed. In patients submitted to radical prostatectomy, PET-PSMA findings were compared to histological analysis of the specimen.

Results: PET-PSMA results of 57 patients were gathered, with $77.2 \%(n=44)$ having performed CT scan or bone scan (BS) prior to PET-PSMA. Prostate cancer management strategy was changed in $61.4 \%(n=27)$, when PET-PSMA was performed following CT and BS. BS and CT results were consistent with PET-PSMA in $43.2 \%$ and $44.8 \%$, respectively. In 30 cases, a curative strategy was used based on PET-PSMA findings. PET-PSMA revealed a negative predictive value of $95.2 \%$ in 23 patients submitted to radical prostatectomy with bilateral pelvic lymphadenectomy. Prostate SUV values on preoperative PET-PSMA correlated with initial PSA, ISUP grade, PC risk staging and presence of extraprostatic lesions.

Conclusions: PET-PSMA is a key element for prostate cancer staging and management, with high diagnostic accuracy. More prospective studies need to be implemented to determine its role as a first-line staging tool.

Keywords: PET-CT, prostate cancer, specificity and sensitivity, metastasis, lymphatic metastases, bone metastases

\section{Introduction}

Accurate staging of prostate cancer (PC) is essential to correctly manage therapeutic strategies with curative intent.

For the clinical staging of PC, current guidelines still recommend a cross-sectional abdominopelvic imaging study for lymph node (LN) evaluation - such as computed tomography (CT) - and a bone scan (BS) for bone metastasis evaluation. These recommendations are however limited to intermediate and high-risk PC. Both imaging modalities have low sensitivity for lesion detection, with a sensitivity of $40 \%$ for LN detection on $\mathrm{CT}^{1}$ and a sensitivity of $80 \%$ for evaluation of bone metastasis in $\mathrm{BS}$ at patient level. ${ }^{2}$ Sensitivities are even lower for PSA values under $10 \mathrm{ng} / \mathrm{mL}$.
Correspondence: João P Lima

Urology and Renal Transplantation

Department, Coimbra University Hospital

Center, Praceta R. Prof. Mota Pinto,

Coimbra, 3004-56I, Portugal

Email joaopedrosolima@gmail.com 
We are currently facing a trend towards replacing standard of care staging modalities with nuclear medicine imaging techniques such as PET/CT with [68Ga]PSMA targeting radiopharmaceuticals (PET-PSMA). ${ }^{3}$ PET-PSMA demonstrates both higher sensitivity and specificity for LN and bone metastasis detection. For LN detection PETPSMA showed per-patient sensitivity of $75-85 \%$ and specificity of $97-99 \%,{ }^{4-6}$ while for bone metastasis detection sensitivity and specificity were, respectively, $96 \%$ and 99.1\%. ${ }^{5}$ In fact, the latest European guidelines already focus on the growing importance of PET-PSMA. ${ }^{7}$ More studies are needed to determine whether the detection of lower volume metastatic disease will alter therapeutic strategies and other endpoints, but preliminary results from the first prospective randomized study (proPSMA) seem to point in that direction. ${ }^{4}$

There are already some reports on the impact of PETPSMA on therapeutic management of PC, with reports of $23 \%$ change on the management of PC when PET-PSMA was used in the primary staging setting, and an even higher impact of PET-PSMA on the management of biochemical recurrence. ${ }^{4,8}$

The high sensitivity for LN disease detection could assist on a better selection of cases who might benefit from bilateral pelvic lymphadenectomy. Yaxley et al studied the predictive value of preoperative PET-PSMA on LN histological metastasis, concluding that by omitting pelvic lymphadenectomy based on a negative preoperative PET-PSMA, one would miss LN disease in $20 \%$ of men. ${ }^{9}$ However, data from the proPSMA study ${ }^{4}$ and from the study of Kulkarni et al ${ }^{10}$ showed higher negative predictive values for PET-PSMA, missing LN disease in 5\% and $12.5 \%$, respectively.

\section{Materials and Methods}

We performed a cross-sectional study based on the experience with PET-PSMA in our center. Eligible patients had a PET-PSMA performed from $01 / 01 / 2016$ to $15 / 03 / 2020$, with PC diagnosed based on a prostate biopsy and with PSA values $>10 \mathrm{ng} / \mathrm{mL}$ and/or ISUP $\geq 2$ (intermediate or high-risk PC). Data regarding sociodemography, clinical presentation, initial PSA, prostate biopsy histology and treatment were collected. Patient data was anonymized and maintained with confidentiality throughout the study.

A dose of $2 \mathrm{MBq} / \mathrm{Kg}$ locally synthetized [68Ga] PSMA-11 was administered 60 minutes prior to wholebody PET/CT acquisition (Siemens Biograph, Siemens Healthcare, Gemini GXL Philips, Philips). Resulting images were then independently interpreted by two nuclear medicine physicians. Prostate SUV values were compared to clinical findings.

Patients were divided into two groups, one comprising patients who performed PET-PSMA alone and another consisting of those who also performed BS or CT. Patients who performed imaging modalities other than PET-PSMA were further studied, regarding whether therapeutic management changed with PET-PSMA.

All patients submitted to radical prostatectomy with bilateral pelvic lymphadenectomy had their histopathological results compared to PET-PSMA findings (Figure 1).

Statistical analysis was performed using SPSS Statistics version 23 , with the use of chi-square test for group comparisons.

\section{Results}

We gathered data on 57 patients, with a mean age of 67.91 \pm 6.59 years. Mean PSA at presentation was $37.73 \pm 68.07$ $\mathrm{ng} / \mathrm{mL}$ and median ISUP grade was 3 . The frequency of intermediate and high-risk PC was, respectively, 28 (49.1\%) and 29 (50.9\%). Thirteen patients performed only PET-PSMA for PC staging, while the remaining 44 performed, in addition to PET-PSMA, BS ( $\mathrm{n}=15)$, CT scan $(n=7)$ or both BS and CT $(n=22)$. Reasons for requesting PET-PSMA were confirmation of solitary or undefined lesions on $\mathrm{BS} / \mathrm{CT}$ in seventeen patients (29.8\%) and absence of extraprostatic disease on BS/CT despite PSA values greater than $50 \mathrm{ng} / \mathrm{mL}$ in seven patients (12.3\%). In the remaining twenty cases (35.1\%) PETPSMA was requested based on physician opinion alone. Mean follow-up after initial treatment was $10.48 \pm 9.64$ months (Table 1).

Considering the group of patients who performed imaging studies other than PET-PSMA $(\mathrm{n}=44)$, staging remained the same after PET-PSMA in 17 cases (38.6\%), while in the remaining $27(61.4 \%)$ PC was re-staged as local disease in 20 cases (45.5\%) and as metastatic disease in $7(15.9 \%)$. Management of the re-staged patients shifted to therapy with curative intent and systemic therapy, respectively. Regarding the 22 patients who performed both BS and CT, PET-PSMA altered PC staging in 14 patients $(63.6 \%)$, with re-staging as local disease in 11 $(52.4 \%)$ and as metastatic disease in $3(14.3 \%)$ (Figure 2).

We also compared the presence or absence of bone lesions on BS and LN on CT versus its detection on PET-PSMA. Of the 37 patients who performed BS, 17 (45.9\%) had lesions that were not detected with PET-PSMA and four $(10.8 \%)$ had 


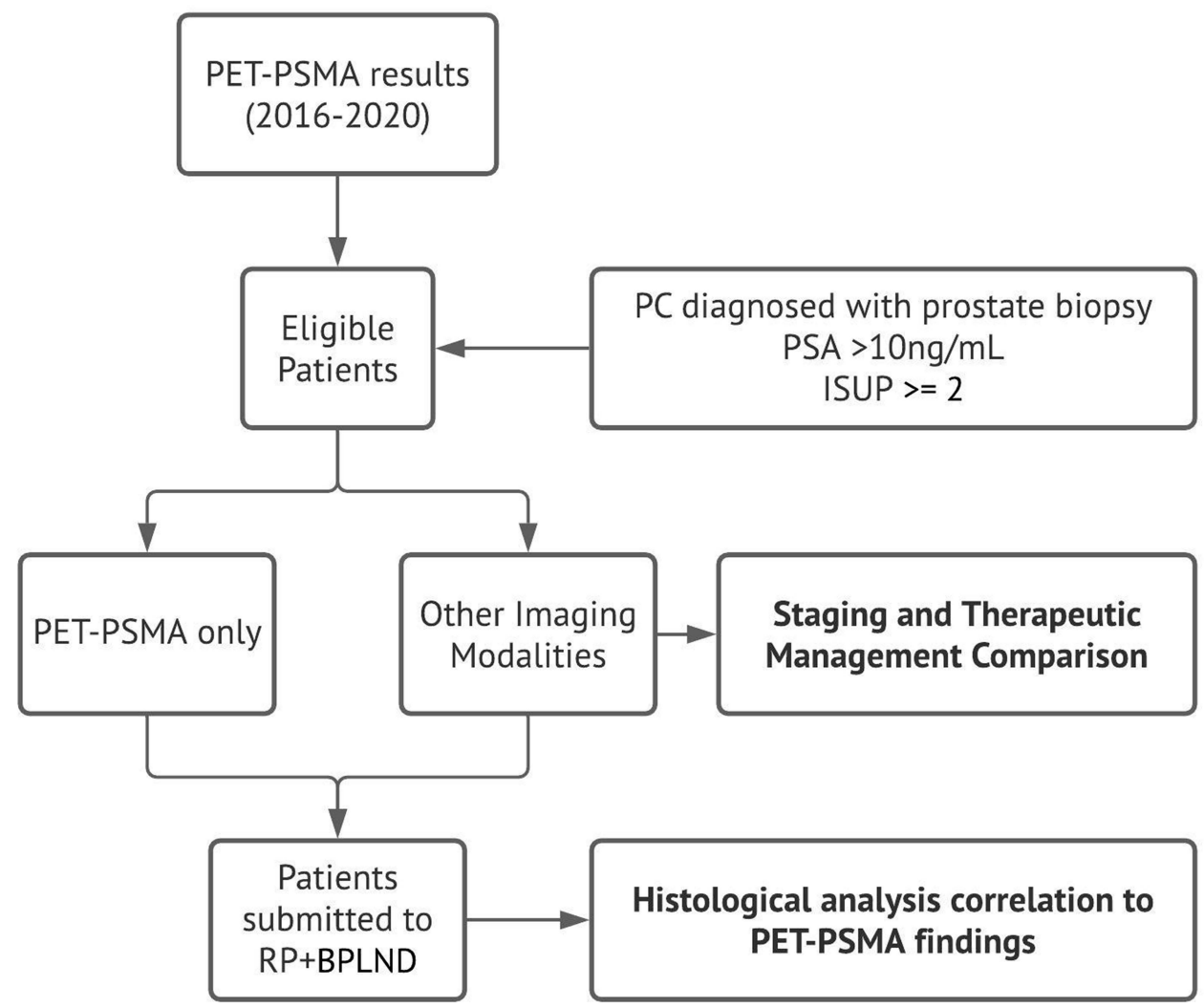

Figure I Study design.

Abbreviations: PET-PSMA, [68Ga] PSMA; PSA, Prostate Specific Antigen; ISUP, International Society of Urological Pathology grading; PC, prostate cancer; RP, radical prostatectomy; BPLND, bilateral lymphadenectomy.

no lesions in BS but were positive for metastization in PET-

PSMA. The remaining 16 (43.2\%) had BS results congruent with PET-PSMA findings. Regarding the 29 patients who

Table I Demographic and Clinical Results

\begin{tabular}{|c|c|c|}
\hline \multicolumn{3}{|l|}{ Population $(n=57)$} \\
\hline Age (years) & \multicolumn{2}{|l|}{$67.91 \pm 6.59$} \\
\hline PSA (ng/mL) & \multicolumn{2}{|l|}{$37.73 \pm 68.07$} \\
\hline \multirow[t]{5}{*}{ ISUP } & I & $3(5.3 \%)$ \\
\hline & 2 & $20(35.1 \%)$ \\
\hline & 3 & 23 (40.4\%) \\
\hline & 4 & $8(14.0 \%)$ \\
\hline & 5 & $3(5.3 \%)$ \\
\hline \multirow[t]{2}{*}{ PC Risk } & Intermediate risk & $28(49.1 \%)$ \\
\hline & High risk & $29(50.9 \%)$ \\
\hline \multirow[t]{4}{*}{ Imaging } & PET-PSMA & $13(22.8 \%)$ \\
\hline & PET-PSMA \& BS & 15 (26.3\%) \\
\hline & PET-PSMA \& CT & 7 (I2.3\%) \\
\hline & PET-PSMA \& BS \& CT & 22 (38.6\%) \\
\hline Follow-up (months) & & $10.48 \pm 9.64$ \\
\hline
\end{tabular}

Abbreviations: PC, Prostate Cancer; PET-PSMA, [68Ga] PSMA; BS, bone scan; CT, computed tomography; ISUP, International Society of Urological Pathology grading; PSA, prostate specific antigen. performed CT before PET-PSMA, 12 (41.4\%) had suspected lymph node disease, not confirmed with PET-PSMA, while 4 (13.8\%) had the opposite findings, no lymph node disease on CT but with lymph nodes with PSMA uptake in PET-PSMA. The remainder ( $\mathrm{n}=13,44.8 \%$ ) had PET-PSMA findings comparable to CT results (Tables 2 and 3).

Overall, 21 patients (36.8\%) received either androgendeprivation therapy (ADT) or chemotherapy with Docetaxel, while 36 patients $(63.2 \%)$ were submitted to treatment with curative intent. The latter were submitted to radical prostatectomy (RP) with or without bilateral lymphadenectomy (BPLND) in 25 cases (73.5\%), external beam radiotherapy (EBRT) in six $(17.6 \%)$, and brachytherapy (BT) in the remaining three cases $(8.8 \%)$. Nine patients $(36.0 \%)$ were eventually submitted to adjuvant RT either for seminal vesicles invasion or positive surgical margins.

Twenty-three out of the 25 patients submitted to RP underwent BPLND. Two of them had positive nodes on the preoperative PET-PSMA, final pathology confirming the findings in one but being negative for nodal metastasis in the other. This last patient had undetected postoperative PSA and remains free from biochemical recurrence 


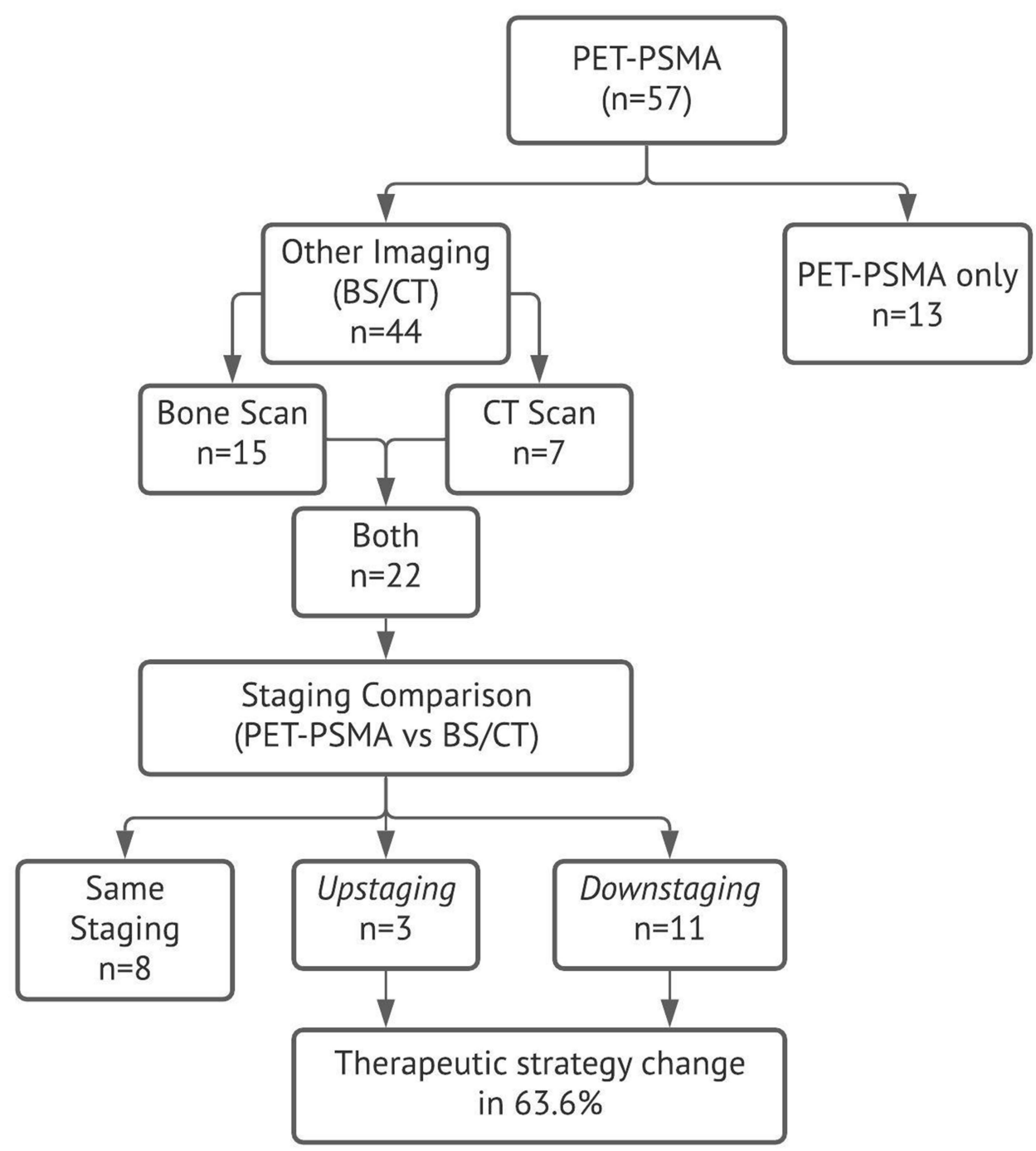

Figure 2 Results.

Abbreviations: PET-PSMA, [68Ga] PSMA; BS, bone scan; CT, computed tomography; Upstaging, Metastatic disease on PET-PSMA, previously reported as local by staging with BS/CT; Downstaging, Local disease on PET-PSMA, previously with suspected secondary lesions on BS/CT.

(BCR). On the other hand, among the 21 patients with negative nodal staging, final pathology revealed metastasis in one, being negative in the remaining twenty.

Table 2 Difference in Staging Comparing PET-PSMA with Standard of Care Imaging

\begin{tabular}{|l|l|r|r|r|}
\hline \multicolumn{2}{|c|}{ n (\%) } & \multicolumn{2}{|r|}{ PET-PSMA } & \multirow{2}{*}{ Total } \\
\cline { 3 - 4 } \multicolumn{2}{|c|}{} & Local & Systemic & \\
\hline $\begin{array}{l}\text { BS and CT } \\
(n=2 I)\end{array}$ & Local & $0(0.0 \%)$ & $3(14.2 \%)$ & $3(14.2 \%)$ \\
\cline { 2 - 4 } & Systemic & $11(52.4 \%)$ & $7(33.3 \%)$ & $18(85.7 \%)$ \\
\hline Total & $11(52.4 \%)$ & $10(47.6 \%)$ & \\
\hline
\end{tabular}

Abbreviations: PET-PSMA, [68Ga] PSMA; BS, bone scan; CT, computed tomography.
Pathological findings were consistent with preoperative PET-PSMA in 21 (91.3\%), while identifying lymph node involvement undetected in PET-PSMA in the one patient $(4.3 \%)$, with a specificity and negative predictive value of $95.2 \%$. Sensitivity and positive predictive values were only $50 \%$, however the extremely low number of positive cases $(\mathrm{n}=2)$, both in PET-PSMA and histopathology adds a very high degree of statistical uncertainty, rendering them unreliable (Table 4).

Regarding all patients with local disease on PET-PSMA staging and who received curative treatment (mean follow-up of $10.48 \pm 9.64$ months, with minimum and maximum followups of two and thirty-six months, respectively), 27 (90.0\%) remained with undetectable PSA at last follow-up, two 
Table 3 Per-Patient Analysis of Lesions in BS and CT versus PETPSMA

\begin{tabular}{|c|c|c|c|c|}
\hline \multicolumn{2}{|l|}{ n (\%) } & \multicolumn{2}{|c|}{$\begin{array}{c}\text { Bone Lesions on PET- } \\
\text { PSMA }\end{array}$} & \multirow[t]{2}{*}{ Total } \\
\hline & & No & Yes & \\
\hline \multirow{2}{*}{$\begin{array}{l}\text { Bone lesions on BS } \\
(n=37)\end{array}$} & No & 14 (37.8\%) & 4 (10.8\%) & \multirow{2}{*}{$\begin{array}{l}18(48.6 \%) \\
19(51.4 \%)\end{array}$} \\
\hline & Yes & 17 (45.9\%) & 2 (5.4\%) & \\
\hline \multicolumn{2}{|l|}{ Total } & $3 \mathrm{I}(83.8 \%)$ & $6(16.2 \%)$ & \\
\hline \multirow{2}{*}{\multicolumn{2}{|c|}{ n (\%) }} & \multicolumn{2}{|c|}{$\begin{array}{l}\text { LN Disease on PET- } \\
\text { PSMA }\end{array}$} & \multirow[t]{2}{*}{ Total } \\
\hline & & No & Yes & \\
\hline \multirow{2}{*}{$\begin{array}{l}\text { LN disease on CT } \\
(n=29)\end{array}$} & No & 4 (13.8\%) & 4 (13.8\%) & \multirow{2}{*}{$\begin{array}{l}8(27.6 \%) \\
21(72.4 \%)\end{array}$} \\
\hline & Yes & 12 (4I.4\%) & 9 (31.0\%) & \\
\hline \multicolumn{2}{|l|}{ Total } & $16(55.2 \%)$ & $13(44.8 \%)$ & \\
\hline
\end{tabular}

Abbreviations: PET-PSMA, [68Ga] PSMA; BS, bone scan; CT, computed tomography; LN disease, lymph node disease.

(6.67\%) suffered a biochemical recurrence and one (3.33\%) was lost to follow-up.

When comparing prostate maximum standardized uptake values (pSUVmax) obtained on PET (not reported in four patients due to overestimation caused by very intense physiologic bladder activity) between patients with localized disease versus metastatic disease, we identified that pSUVmax values correlate with initial PSA, ISUP grade, PC risk staging, presence of secondary lesions and with the endpoint of therapy with curative intent, with statistical significance. pSUVmax did not correlate with age, local pathological stage on radical prostatectomy specimens (T stage) or surgical margins (Table 5).

\section{Discussion}

PET-PSMA is rapidly proving its ability to overcome other imaging modalities for the staging of PC. This is supported by a systematic review on the use of PET-PSMA for initial

Table 4 Pelvic Node Sampling Analysis of Patients Submitted to Pelvic Lymphadenectomy

\begin{tabular}{|l|r|r|r|r|}
\hline \multirow{2}{*}{ n (\%) } & \multicolumn{2}{|}{$\begin{array}{r}\text { LN Disease on } \\
\text { PET-PSMA }\end{array}$} & \multirow{2}{*}{ Total } \\
\cline { 2 - 4 } & & No & Yes & \\
\hline LN disease on & No & $20(87.0 \%)$ & I (4.3\%) & $21(91.3 \%)$ \\
Histopathology (N=23) & Yes & $1(4.3 \%)$ & I (4.3\%) & $2(8.7 \%)$ \\
\hline Total & $21(91.3 \%)$ & $2(8.7 \%)$ & \\
\hline
\end{tabular}

Abbreviations: PET-PSMA, [68Ga] PSMA; LN disease, lymph node disease. staging of PC 11, and from a prospective, randomized, multicenter study on this subject (proPSMA). ${ }^{4}$

Current guidelines still advocate BS and a crosssectional abdominopelvic imaging study for initial staging of patients with intermediate and high-risk $\mathrm{PC}^{7}$ even though sensitivity for CT for the detection of lymph node disease range from 38 to $42 \%,{ }^{1,4,11}$ compared with sensitivity of $85-99 \%$ for PET-PSMA. ${ }^{4,12}$

Most studies, including ours, compared conventional imaging with PET-PSMA in patients with intermediate and high-risk PC, while the proPSMA study gathered information on 339 patients with high-risk PC alone. Roach et al reported changes in patient management in $21 \%$ of patients, ${ }^{8}$ while in the proPSMA study $28 \%$ of patients with high-risk PC suffered treatment change with PETPSMA, with $14 \%$ being upstaged with PET-PSMA. ${ }^{4}$ In our study, management strategy was changed in $61.4 \%$ of patients, with $45.5 \%$ directed to curative intent and $15.9 \%$ to systemic treatment. Being a retrospective study, this difference may be attributed to a selection bias, where PET-PSMA was mainly performed when radiological findings were not consistent with clinical suspicion. This selection bias, together with a small sample of patients, did not allow for subgroup analysis, which would have been important for understanding in which patients PETPSMA was most beneficial.

There are few studies comparing PET-PSMA findings with pathological sampling of pelvic nodes, with Herlemann et al first reporting in a sampling of 34 patients a sensitivity, specificity, positive predictive value (PPV) and negative predictive value (NPV) of $91 \%, 67 \%, 83 \%$ and $80 \%$ respectively. ${ }^{6}$ More recently Kulkarni et al reported an NPV of $87.5 \%$ from a cohort of 51 patients, while in the proPSMA study, in the subgroup of patients who performed PET-PSMA and RP with BPLND $(n=145)$, only $5 \%$ of patients with a negative PET-PSMA had lymph node disease. These latter results are more in line with our reported NPV of $95.2 \%$, with only $5 \%$ of missed lymph node disease on preoperative PET-PSMA.

We also identified a correlation between higher pSUVmax values on PET-PSMA, higher ISUP grades and metastatic disease. This has been previously reported by Demirci et al, ${ }^{13}$ and indicates that pSUVmax may predict clinical significant $\mathrm{PC}$, with a clear difference in mean pSUVmax values between ISUP grades 1-2 and ISUP grades 3-5. pSUVmax has also been inversely correlated with $\mathrm{ADC}$ in multiparametric magnetic resonance imaging (mpMRI), suggesting a combination of both PET 
Table 5 PET-PSMA pSUVmax Correlation with Clinical Findings

\begin{tabular}{|c|c|c|c|c|}
\hline & & $\mathbf{n}$ & Mean pSUVmax & $\mathbf{p}$ \\
\hline Age & & & & 0.635 \\
\hline \multirow[t]{2}{*}{ Initial PSA } & $<20 \mathrm{ng} / \mathrm{mL}$ & 30 & $9.66( \pm 7.00)$ & \multirow[t]{2}{*}{0.001} \\
\hline & $>20 \mathrm{ng} / \mathrm{mL}$ & 22 & $18.5 \mid( \pm 10.44)$ & \\
\hline \multirow[t]{2}{*}{ ISUP } & $\leq 2$ & 20 & $9.06( \pm 7.5 \mathrm{I})$ & \multirow[t]{2}{*}{0.011} \\
\hline & $>2$ & 26 & $15.86( \pm 9.82)$ & \\
\hline \multirow[t]{2}{*}{ Metastatic Disease } & No & 32 & $10.29( \pm 7.33)$ & \multirow[t]{2}{*}{0.004} \\
\hline & Yes & 21 & $15.89( \pm 10.82)$ & \\
\hline \multirow[t]{2}{*}{ Therapy with Curative Intent } & No & 19 & $18.85( \pm 10.78)$ & \multirow[t]{2}{*}{0.001} \\
\hline & Yes & 34 & $10.19( \pm 7.24)$ & \\
\hline Pathological Staging $(\mathrm{T})$ & & & & 0.993 \\
\hline \multirow[t]{2}{*}{ Surgical Margins (R) } & RO & 16 & $8.45( \pm 7.74)$ & \multirow[t]{2}{*}{0.277} \\
\hline & RI & 5 & $12.80( \pm 6.96)$ & \\
\hline
\end{tabular}

Abbreviations: PET-PSMA, [68Ga] PSMA; pSUVmax, prostate maximum standardized uptake values; PSA, prostate specific antigen; ISUP, International Society of Urological Pathology grading.

and mpMRI for biopsy targeting. ${ }^{13}$ Kulkarni et al also identified a correlation on univariate analysis between pathological T-staging and pSUVmax and the presence of lymphatic metastization, with no patient with a pSUVmax less than 5 having lymph node disease. ${ }^{10}$ These findings are consistent with our data, where in the subgroup of 23 patients with lymph node disease, only one had a pSUVmax inferior to 5 .

\section{Conclusions}

This study supports the use of PET-PSMA for the initial staging of intermediate and high-risk PC, having changed initial staging in over $60 \%$ of cases and allowing for a better-informed clinical decision. In patients submitted to RP, pathology findings were very consistent with PETPSMA results, with a specificity and NPV of $95.2 \%$, which confirms its high diagnostic accuracy.

Overall, PET-PSMA is set to be an increasingly key element for PC staging and management definition. More prospective studies are needed, however, to determine whether PET-PSMA should be a first line staging tool and to define whom will benefit the most from it.

\section{Ethics Statement}

This study was performed in accordance with the Declaration of Helsinki. Data was gathered from the institution database with granted permission. Patient data was anonymized and maintained with confidentiality throughout the study.

\section{Author Contributions}

João Lima, João Carvalho, Vasco Quaresma, Edgar Tavares da Silva, Paulo Azinhais, Arnaldo Figueiredo, Rodolfo Silva and Gracinda Costa contributed significantly to the conception of the study, acquisition of data, analysis of the results and revision of the manuscript. All authors read and approved the final version of the manuscript. All authors have agreed to submit this study to Research and Reports in Urology and take responsibility and are accountable for the contents of the article.

\section{Disclosure}

The authors certify that there are no conflicts of interest with any financial organization regarding the material discussed in the manuscript.

\section{References}

1. Hövels AM, Heesakkers RAM, Adang EM, et al. The diagnostic accuracy of CT and MRI in the staging of pelvic lymph nodes in patients with prostate cancer: a meta-analysis. Clin Radiol. 2008;63 (4):387-395. doi:10.1016/j.crad.2007.05.022

2. Shen G, Deng H, Hu S, Jia Z. Comparison of choline-PET/CT, MRI, SPECT, and bone scintigraphy in the diagnosis of bone metastases in patients with prostate cancer: a meta-analysis. Skeletal Radiol. 2014;43 (11):1503-1513. doi:10.1007/s00256-014-1903-9

3. Haran C, McBean R, Parsons R, Wong D. Five-year trends of bone scan and prostate-specific membrane antigen positron emission tomography utilization in prostate cancer: a retrospective review in a private centre. J Med Imaging Radiat Oncol. 2019;63(4):495-499. doi:10.1111/1754-9485.12885

4. Esen T, Kılıç M, Seymen H, et al. Can Ga-68 PSMA PET/CT replace conventional imaging modalities for primary lymph node and bone staging of prostate cancer? Eur Urol Focus. 2020;6:218-220. doi:10.1016/j.euf.2019.05.005 
5. Mottet N, Bellmunt J, Briers E, et al. WT members of the E- e- e SIOG PCGP. EAU - ESTRO - ESUR - SIOG guidelines on prostate cancer. 2020.

6. Hofman MS, Lawrentschuk N, Francis RJ, et al. Prostate-specific membrane antigen PET-CT in patients with high-risk prostate cancer before curative-intent surgery or radiotherapy (proPSMA): a prospective, randomised, multi-centre study. Lancet. 2020;6736 (20):1-9. doi:10.1016/s0140-6736(20)30314-7

7. Roach PJ, Francis R, Emmett L, et al. The impact of (68) Ga-PSMAPET/CT on management intent in prostate cancer: results of an Australian prospective multicenter study. J Nucl Med. 2018;59 (1):82-88. doi:10.2967/jnumed.117.197160

8. Yaxley JW, Raveenthiran S, Nouhaud FX, et al. Outcomes of primary lymph node staging of intermediate and high risk prostate cancer with (68) Ga-PSMAPositron emission tomography/computerized tomography compared to histological correlation of pelvic lymph node pathology. J Urol. 2019;201(4):815-820. doi:10.1097/JU.0000000000000053

9. Kulkarni SC, Sundaram PS, Padma S. In primary lymph nodal staging of patients with high-risk and intermediate-risk prostate cancer, how critical is the role of Gallium-68 prostate-specific membrane antigen positron emission tomography-computed tomography? Nucl Med Commun. 2020;41(2):139-146. doi:10.1097/MNM.0000000 000001110
10. Petersen LJ, Zacho HD. PSMA PET for primary lymph node staging of intermediate and high-risk prostate cancer: an expedited systematic review. Cancer Imaging. 2020;20(1):1-8. doi:10.1186/s40644-0200290-9

11. Herlemann A, Wenter V, Kretschmer A, et al. 68Ga-PSMA positron emission tomography/computed tomography provides accurate staging of lymph node regions prior to lymph node dissection in patients with prostate cancer. Eur Urol. 2016;70(4):553-557. doi:10.1016/j. eururo.2015.12.051

12. Demirci E, Kabasakal L, Şahin OE, et al. Can SUVmax values of Ga-68-PSMA PET/CT scan predict the clinically significant prostate cancer? Nucl Med Commun. 2019;40(1):86-91. doi:10.1097/ MNM.0000000000000942

13. Uslu-Beşli L, Bakır B, Asa S, et al. Correlation of SUVmax and apparent diffusion coefficient values detected by Ga-68 PSMA PET/ MRI in primary prostate lesions and their significance in lymph node metastasis: preliminary results of an on-going study. Mol Imaging Radionucl Ther. 2019;28(3):104-111. doi:10.4274/mirt. galenos.2019.63825
Research and Reports in Urology

\section{Publish your work in this journal}

Research and Reports in Urology is an international, peer-reviewed, open access journal publishing original research, reports, editorials, reviews and commentaries on all aspects of adult and pediatric urology in the clinic and laboratory including the following topics Pathology, pathophysiology of urological disease; Investigation and
Dovepress

treatment of urological disease; Pharmacology of drugs used for the treatment of urological disease. The manuscript management system is completely online and includes a very quick and fair peer-review system, which is all easy to use. Visit http://www.dovepress.com/ testimonials.php to read real quotes from published authors. 